\title{
Yield Parameters and Yield of Aerobic Rice (Oryza sativa) as Influenced by Different Seeding Methods and Weed Control Measures
}

\author{
C.H. Prashanthi ${ }^{1 *}$, P. Laxminarayana ${ }^{1}$, G.E.C.H. Vidyasagar ${ }^{1}$ and \\ S. Harish Kumar Sharma \\ ${ }^{1}$ Department of Agronomy, College of Agriculture, Telangana, India \\ ${ }^{2}$ Department of Soil Science and Agricultural Chemistry, Professor Jayashankar Telangana State \\ Agricultural University, Rajendranagar, Hyderabad - 500 030, Telangana, India \\ *Corresponding author
}

\section{A B S T R A C T}

Keywords

Aerobic rice, Yield parameters and yield.

Article Info

Accepted:

26 June 2017

Available Online:

10 July 2017
Field investigation on evaluation of pre and post emergent herbicides on yield parameters and yield of aerobic rice was conducted during kharif 2014 at agricultural college Farm, Rajendranagar, Hyderabad. The experiment consisted of combination of pre and post emergence herbicides alone, in combination with hand weeding and hand weedings at 20, $40 \&$ 60 DAS and un-weeded check. The results revealed hand weeding at 20, 40 and 60 DAS recorded significantly higher growth and yield components viz., no. of panicles $/ \mathrm{m}^{2}$, test weight, panicle length, panicle weight, grain yield, straw yield and higher weed control efficiency which was at par with Pretilachlor $0.75 \mathrm{~kg}$ ai ha ${ }^{-1}$ as PE fb Azimsulfuron 35g.ai ha ${ }^{-1}+$ Cyhalofop butyl $75 \mathrm{~g}$. ai ha ${ }^{-1}$ as PoE 15-20 DAS followed by hand weeding at 50 DAS under both the seeding methods.

\section{Introduction}

Rice is the world's second most important cereal crop and staple food for about three billion people across the globe and the demand for food continue to increase as the population is increasing at faster rate (Carriger and Valle, 2007).

Direct sown aerobic rice suffers more due to weed menace as the weeds and rice compete for growth factors together and weeds cause yield loss between 30 and 98 percent (Oerke and Dehne, 2004). Pretilachlor as preemergence herbicide, is not effective against grasses and sedges while azimsulfuron was the new selective, post-emergence herbicide was found to be most efficient against sedges and broadleaf weeds and less effective against grassy weeds.

However, sequential application of herbicides along with one hand weeding was reported to be more effective than application of herbicides alone, hence the present investigation was undertaken to study the efficacy of sequential application of pre and post emergence herbicides. 


\section{Materials and Methods}

Field experiment was carried out during kharif, 2014 at College Farm, Professor Jayashankar Telangana State Agricultural University, Hyderabad to evaluate the efficacy of sequential application of herbicides in different seeding methods in sandy loam soil. The experiment was conducted in factorial RBD with a plot size of $4 \times 4 \mathrm{~m}$ with three replications. Factor 1 includes seeding methods, broadcasting $\left(S_{1}\right)$ and line sowing $\left(\mathrm{S}_{2}\right)$ Factor II includes weed management practices, $\mathrm{T}_{1}$-Pretilachlor $\mathrm{fb}$ metsulfuron methyl + chlorimuron ethyl + cyhalofop butyl at 15-20 DAS, $\mathrm{T}_{2}$-Pretilachlor $\mathrm{fb}$ azimsulfuron + cyhalofop butyl 15-20 DAS, $\mathrm{T}_{3}$-Pretilachlor $0.75 \mathrm{~kg}$ ai $\mathrm{ha}^{-1} \mathrm{fb}$ pyrazosulfuron ethyl + cyhalofop butyl at 15 20 DAS, $\mathrm{T}_{4}$-bispyribac sodium $25 \mathrm{~g}$ ai ha ${ }^{-1} \mathrm{fb}$ 2-4-D $0.5 \mathrm{~kg}$.ai ha ${ }^{-1}$ at 40 DAS, $\mathrm{T}_{5^{-}} \mathrm{T}_{1}$ followed by $\mathrm{HW}$ at $50 \mathrm{DAS}, \mathrm{T}_{6}-\mathrm{T}_{2}$ followed by $\mathrm{HW}$ at $50 \mathrm{DAS}, \mathrm{T}_{7-} \mathrm{T}_{3}$ followed by $\mathrm{HW}$ at $50 \mathrm{DAS}, \mathrm{T}_{8}-\mathrm{T}_{4}$ followed by $\mathrm{HW}$ at $50 \mathrm{DAS}$, $\mathrm{T}_{9^{-}} \mathrm{HW}$ at 20, 40 and 60 DAS, $\mathrm{T}_{10}$-unweeded control. The recommended fertilizer dose was 100-60-40 kg of $\mathrm{N}, \mathrm{P}_{2} \mathrm{O}_{5}$ and $\mathrm{K}_{2} \mathrm{O} \mathrm{ha}^{-1}$ respectively.

\section{Results and Discussion}

Weed flora such as Echinocloa colonam L., Cynodon dactylon L., Eleusine indica among the grasses; Cyperus rotundus $\mathrm{L}$ among the sedges and Eclipta alba L., Commelina bengalensis L., Ipomoea purpurea, Alternanthera sessillis, Physalis minima, Bacopa monnieri, Cyanotis cristata, Corchorus, Phyllanthus niruri, Ageratum conyzoides, among the broad-leaved weeds, were found to be predominant weeds in aerobic rice. Herbicidal treatments significantly influenced the grain yield. The higher WCE (95.5) was recorded with $\left(\mathrm{T}_{9}\right)$ hand weeding thrice at $60 \mathrm{DAS}$. Which was at par with $\mathrm{T}_{6}$ - Pretilachlor fb azimsulfuron + cyhalofop butyl 15-20 DAS fb HW at 50 DAS with regard to WCE $(95.3 \%)$ and grain yield $\left(3218 \mathrm{~kg} \mathrm{ha}^{-1}\right)$ indicating that weeds are controlled efficiently with sequential application of herbicides resulted in higher grain yield. Grain yield was influenced by the interaction effect of both seeding methods and weed management practices. Hand weeding recorded significantly higher yield under line sowing method.

Among seeding methods the higher grain yield was recorded with line sowing $\left(\mathrm{S}_{2}\right)$ $\left(3161.0 \mathrm{~kg} \mathrm{ha}^{-1}\right)$ than the broadcasting $\left(\mathrm{S}_{1}\right)$ $\left(2366.0 \mathrm{~kg} \mathrm{ha}^{-1}\right)$ method which might be due to the maintenance of less weed population and higher weed control efficiency.

\section{Weed control efficiency}

Among the seeding methods, significantly higher weed control efficiency was observed in the line sowing $\left(\mathrm{S}_{2}\right)$ than broadcasting $\left(\mathrm{S}_{1}\right)$.

At maturity, the highest weed control efficiency was recorded with hand weeding $\mathrm{T}_{9}$ and it was significantly superior to all other treatments. Among weed management practices tried, higher weed control efficiency was observed with $\mathrm{T}_{6}(41.45 \%)$ which was at par with $\mathrm{T}_{5}, \mathrm{~T}_{7}, \mathrm{~T}_{8}(40.55,39.7$ and 39.15 respectively) and these treatments were significantly superior over $\mathrm{T}_{1}, \mathrm{~T}_{2}, \mathrm{~T}_{3}$ and $\mathrm{T}_{4}$ $(29.75,30.25,29.7$ and 29.25 respectively). This could probably due to effective control of grasses, with pre emergence herbicide application and sedges and broad leaf weeds by the application of post emergence herbicides. The higher weed control efficiency $\left(T_{9}\right)$ was due to complete suppressal of weeds particularly during critical stage from 20 to 40 DAS, thereby keeping crop weed free, and thus avoiding weed competition during critical stages, resulting in better growth (Table 1). 
This could be the result of less weed competition for growth factors during critical period of growing due to effective weed control as evident from higher WCE. These results were in agreement with Walia et al., (2008b). Severe weed infestation in unweeded control resulted in more competition for sunlight, nutrients, moisture and space inhibiting the crop growth.

\section{Yield parameters}

Test weight, panicle length and panicle weight were significantly influenced by method of sowing and weed management practices but not by their interaction.

\section{No. of panicles $\mathbf{m}^{-2}$}

Data pertaining to yield attributes as influenced by seeding methods and weed management practices is presented in table 2 . Close observation of data indicated that among seeding methods line sowing produced significantly higher number of panicles $\mathrm{m}^{-2}$ (257.0) compared with broadcasting (234.8) similar results reported by Anwar et al., (2011).

With regard to weed management practices, the highest number of panicles $\mathrm{m}^{-2}$ were produced with hand weeding $\left(\mathrm{T}_{9}\right)$ and it was at par with $\mathrm{T}_{6}(262.0), \mathrm{T}_{5}(257.5), \mathrm{T}_{7}(254.5)$, $\mathrm{T}_{8}$ (256.0) and $\mathrm{T}_{2}$ (269.1) which are significantly superior over rest of the weed management practices. The next best weed management practice in producing higher number of panicles was $T_{1}(250.5)$ which in turn on par with $\mathrm{T}_{3}(249)$ and $\mathrm{T}_{4}$ (248.6). More number of panicles at hand weeding might be due to effective control of weeds throughout the crop growth period, could resulted in better translocation of photosynthates from source to sink. Similar results were reported by Saha (2005), Thimme Gowda et al., (2009).

Table.1 Weed control efficiency (\%) of aerobic rice at 60 DAS and at harvest as influenced by Weed management practices under different seeding methods

\begin{tabular}{|c|c|c|c|c|c|c|c|}
\hline \multirow[t]{3}{*}{$\mathbf{T}$} & \multirow[t]{3}{*}{ Weed management practices } & \multicolumn{6}{|c|}{ Seeding methods } \\
\hline & & \multicolumn{3}{|c|}{60 DAS } & \multicolumn{3}{|c|}{ Harvest } \\
\hline & & $\mathbf{S}_{1}$ & $\mathbf{S}_{\mathbf{2}}$ & Mean & $\mathbf{S}_{1}$ & $\mathbf{S}_{2}$ & Mean \\
\hline $\mathbf{T}_{1}$ & $\begin{array}{l}\text { Pretilachlor } 0.75 \mathrm{~kg} \text { ai } \mathrm{ha}^{-1} \text { as PE fb } \\
\text { Metsulfuron methyl }+ \text { Chlorimuron ethyl } 4 \mathrm{~g} \text {. } \\
\text { ai ha }{ }^{-1} \text { as PoE }+ \text { Cyhalofop butyl } 75 \mathrm{~g} \text {. ai ha }{ }^{-1} \\
\text { as PoE at } 15-20 \text { DAS. }\end{array}$ & 43.1 & 43.3 & 43.2 & 29.2 & 30.3 & 29.75 \\
\hline $\mathbf{T}_{2}$ & $\begin{array}{l}\text { Pretilachlor } 0.75 \text { kg ai } \text { ha }^{-1} \text { as PE fb } \\
\text { Azimsulfuron 35g.ai ha }{ }^{-1}+\text { Cyhalofop butyl } 75 \\
\text { g. ai ha }{ }^{-1} \text { as PoE 15-20 DAS. }\end{array}$ & 42.8 & 44.2 & 43.5 & 29.6 & 30.9 & 30.25 \\
\hline $\mathbf{T}_{3}$ & $\begin{array}{l}\text { Pretilachlor } 0.75 \mathrm{~kg} \text { ai } \mathrm{ha}^{-1} \text { as } \mathrm{PE} \mathrm{fb} \\
\text { Pyrazosulfuron ethyl } 20 \text { g.ai ha }{ }^{-1}+\text { Cyhalofop } \\
\text { butyl } 75 \text { g. ai ha- }{ }^{-1} \text { as PoE at } 15-20 \text { DAS. }\end{array}$ & 42.6 & 43.4 & 43.0 & 29.0 & 30.4 & 29.7 \\
\hline $\mathbf{T}_{4}$ & $\begin{array}{l}\text { Bispyribac sodium } 25 \mathrm{~g} \text { ai ha }{ }^{-1} \text { as early PoE fb } \\
\text { 2-4-D } 0.5 \mathrm{~kg} \cdot \mathrm{ai} \mathrm{ha}^{-1} \text { at } 40 \text { DAS. }\end{array}$ & 42.2 & 43.0 & 42.6 & 28.5 & 30.0 & 29.25 \\
\hline $\mathbf{T}_{5}$ & $\mathrm{~T}_{1} \mathrm{fb}$ Hand weeding at 50 DAS. & 94.4 & 95.7 & 95.0 & 39.9 & 41.2 & 40.55 \\
\hline $\mathbf{T}_{6}$ & $\mathrm{~T}_{2}$ fb Hand weeding at $50 \mathrm{DAS}$. & 94.8 & 95.8 & 95.3 & 40.9 & 42.0 & 41.45 \\
\hline $\mathbf{T}_{7}$ & $\mathrm{~T}_{3}$ fb Hand weeding at 50 DAS. & 94.3 & 95.6 & 94.9 & 38.7 & 40.8 & 39.7 \\
\hline $\mathbf{T}_{8}$ & $\mathrm{~T}_{4} \mathrm{fb}$ Hand weeding at 50 DAS. & 94.2 & 95.5 & 94.8 & 37.9 & 40.4 & 39.15 \\
\hline $\mathbf{T}_{9}$ & Hand weeding at $20,40,60$ DAS & 95.2 & 95.9 & 95.5 & 84.4 & 85.8 & 85.1 \\
\hline $\mathbf{T}_{10}$ & Unweeded (control) & 0 & 0 & $\mathbf{0}$ & 0 & 0 & \\
\hline
\end{tabular}


Table. 2 Yield parameters of aerobic rice as influenced by weed management practices Under different seeding methods

\begin{tabular}{|c|c|c|c|c|c|c|c|}
\hline \multirow[t]{3}{*}{$\mathbf{T}$} & \multirow[t]{3}{*}{ Weed management practices } & \multicolumn{6}{|c|}{ Seeding methods } \\
\hline & & \multicolumn{3}{|c|}{ No.of panicles/m2 } & \multicolumn{3}{|c|}{ Test weight (g) } \\
\hline & & $\mathbf{S}_{1}$ & $\mathbf{S}_{2}$ & Mean & $\mathbf{S}_{1}$ & $\mathbf{S}_{\mathbf{2}}$ & Mean \\
\hline $\mathbf{T}_{1}$ & $\begin{array}{l}\text { Pretilachlor } 0.75 \mathrm{~kg} \text { ai ha }{ }^{-1} \text { as PE fb } \\
\text { Metsulfuron methyl }+ \text { Chlorimuron ethyl } \\
4 \text { g. ai ha }{ }^{-1} \text { as PoE }+ \text { Cyhalofop butyl } 75 \\
\text { g. ai ha }{ }^{-1} \text { as PoE at } 15-20 \text { DAS. }\end{array}$ & 254.6 & 246.3 & 250.5 & 17.63 & 20.16 & 18.90 \\
\hline $\mathbf{T}_{2}$ & $\begin{array}{l}\text { Pretilachlor } 0.75 \mathrm{~kg} \text { ai } \mathrm{ha}^{-1} \text { as } \mathrm{PE} \mathrm{fb} \\
\text { Azimsulfuron } 35 \mathrm{~g} \cdot \mathrm{ai} \mathrm{ha}^{-1}+\text { Cyhalofop } \\
\text { butyl } 75 \mathrm{~g} \text {. ai ha }{ }^{-1} \text { as PoE } 15-20 \text { DAS. }\end{array}$ & 280.0 & 258.3 & 269.1 & 17.73 & 20.90 & 19.31 \\
\hline $\mathbf{T}_{3}$ & $\begin{array}{l}\text { Pretilachlor } 0.75 \mathrm{~kg} \text { ai/ha as } \mathrm{PE} \text { fb } \\
\text { Pyrazosulfuron ethyl } 20 \text { g.ai } \mathrm{ha}^{-1}+ \\
\text { Cyhalofop butyl } 75 \text { g. ai ha }{ }^{-1} \text { as PoE at } \\
\text { 15-20 DAS. }\end{array}$ & 243.6 & 254.3 & 249.0 & 16.96 & 20.36 & 18.66 \\
\hline $\mathbf{T}_{4}$ & $\begin{array}{l}\text { Bispyribac sodium } 25 \mathrm{~g} \text { ai } \mathrm{ha}^{-1} \text { as early } \\
\text { PoE fb 2-4-D } 0.5 \mathrm{~kg} \text {.ai ha }{ }^{-1} \text { at } 40 \text { DAS. }\end{array}$ & 246.6 & 250.6 & 248.6 & 16.73 & 20.20 & 18.46 \\
\hline $\mathbf{T}_{5}$ & $\mathrm{~T}_{1} \mathrm{fb}$ Hand weeding at $50 \mathrm{DAS}$. & 229.6 & 285.3 & 257.5 & 20.00 & 21.73 & 20.86 \\
\hline$T_{6}$ & $\mathrm{~T}_{2} \mathrm{fb}$ Hand weeding at $50 \mathrm{DAS}$. & 236.6 & 287.3 & 262.0 & 20.63 & 21.90 & 21.26 \\
\hline $\mathbf{T}_{7}$ & $\mathrm{~T}_{3} \mathrm{fb}$ Hand weeding at $50 \mathrm{DAS}$. & 225.6 & 283.3 & 254.5 & 20.36 & 21.06 & 20.71 \\
\hline $\mathbf{T}_{8}$ & $\mathrm{~T}_{4} \mathrm{fb}$ Hand weeding at $50 \mathrm{DAS}$. & 230.0 & 282.0 & 256.0 & 20.20 & 21.03 & 20.61 \\
\hline $\mathbf{T}_{9}$ & Hand weeding at $20,40,60$ DAS & 251.3 & 288.0 & 269.6 & 20.66 & 22.10 & 21.38 \\
\hline $\mathbf{T}_{10}$ & Unweeded (control) & 149.6 & 134.3 & 142.0 & 18.16 & 18.63 & 18.40 \\
\hline & Mean & 234.8 & 257.0 & & 18.9 & 20.8 & \\
\hline & & SEm \pm & CD & & SEm \pm & CD & \\
\hline & F1 & 2.52 & 7.23 & & 0.18 & 0.54 & \\
\hline & F2 & 5.64 & 16.17 & & 0.42 & 1.21 & \\
\hline & F1 $\times F 2$ & 7.98 & 22.87 & & 0.59 & NS & \\
\hline
\end{tabular}


Table.3 Yield parameters of aerobic rice as influenced by weed management practices Under different seeding methods

\begin{tabular}{|c|c|c|c|c|c|c|c|}
\hline \multirow[t]{3}{*}{$\mathbf{T}$} & \multirow[t]{3}{*}{ Weed management practices } & \multicolumn{6}{|c|}{ Seeding methods } \\
\hline & & \multicolumn{3}{|c|}{ Panicle length (cm) } & \multicolumn{3}{|c|}{ Panicle weight (g) } \\
\hline & & $\mathbf{S}_{1}$ & $\mathbf{S}_{2}$ & Mean & $\mathbf{S}_{1}$ & $\mathbf{S}_{2}$ & Mean \\
\hline $\mathbf{T}_{1}$ & $\begin{array}{l}\text { Pretilachlor } 0.75 \mathrm{~kg} \text { ai } \mathrm{ha}^{-1} \text { as PE fb } \\
\text { Metsulfuron methyl + Chlorimuron ethyl } 4 \\
\text { g. ai ha }{ }^{-1} \text { as PoE + Cyhalofop butyl } 75 \mathrm{~g} \text {. ai } \\
\text { ha }^{-1} \text { as PoE at 15-20 DAS. }\end{array}$ & 15.4 & 16.4 & 15.9 & 1.11 & 1.28 & 1.19 \\
\hline $\mathbf{T}_{2}$ & $\begin{array}{l}\text { Pretilachlor } 0.75 \mathrm{~kg} \text { ai } \mathrm{ha}^{-1} \text { as PE fb } \\
\text { Azimsulfuron } 35 \mathrm{~g} \text {.ai ha }{ }^{-1}+\text { Cyhalofop butyl } \\
75 \text { g. ai ha }{ }^{-1} \text { as PoE } 15-20 \text { DAS. }\end{array}$ & 15.4 & 16.7 & 16.0 & 1.24 & 1.29 & 1.26 \\
\hline $\mathbf{T}_{3}$ & $\begin{array}{l}\text { Pretilachlor } 0.75 \text { kg ai/ha as PE fb } \\
\text { Pyrazosulfuron ethyl } 20 \text { g.ai } \text { ha }^{-1}+ \\
\text { Cyhalofop butyl } 75 \text { g. ai } \text { ha }^{-1} \text { as PoE at } 15- \\
20 \text { DAS. }\end{array}$ & 15.2 & 16.3 & 15.7 & 1.18 & 1.27 & 1.23 \\
\hline $\mathbf{T}_{4}$ & $\begin{array}{l}\text { Bispyribac sodium } 25 \mathrm{~g}^{\text {ai ha }}{ }^{-1} \text { as early PoE } \\
\text { fb 2-4-D } 0.5 \mathrm{~kg} \cdot \mathrm{ai} \mathrm{ha}^{-1} \text { at } 40 \text { DAS. }\end{array}$ & 15.1 & 15.9 & 15.5 & 1.21 & 1.22 & 1.21 \\
\hline $\mathbf{T}_{5}$ & $\mathrm{~T}_{1} \mathrm{fb}$ Hand weeding at $50 \mathrm{DAS}$. & 16.5 & 17.6 & 17.0 & 1.26 & 1.27 & 1.27 \\
\hline$T_{6}$ & $\mathrm{~T}_{2} \mathrm{fb}$ Hand weeding at $50 \mathrm{DAS}$. & 16.8 & 17.8 & 17.3 & 1.28 & 1.40 & 1.34 \\
\hline $\mathbf{T}_{7}$ & $\mathrm{~T}_{3} \mathrm{fb}$ Hand weeding at $50 \mathrm{DAS}$. & 16.3 & 17.5 & 16.9 & 1.22 & 1.35 & 1.28 \\
\hline $\mathbf{T}_{8}$ & $\mathrm{~T}_{4} \mathrm{fb}$ Hand weeding at $50 \mathrm{DAS}$. & 16.7 & 17.4 & 17.0 & 1.22 & 1.31 & 1.26 \\
\hline $\mathbf{T}_{9}$ & Hand weeding at $20,40,60$ DAS & 19.8 & 20.4 & 20.1 & 1.34 & 1.53 & 1.43 \\
\hline $\mathbf{T}_{10}$ & Unweeded (control) & 14.9 & 17.8 & 16.3 & 0.82 & 0.87 & 0.84 \\
\hline & Mean & 16.2 & 17.4 & & 1.19 & 1.28 & \\
\hline & & SEm \pm & CD & & SEm \pm & CD & \\
\hline & F1 & 0.15 & 0.44 & & 0.01 & 0.05 & \\
\hline & F2 & 0.34 & 0.98 & & 0.03 & 0.11 & \\
\hline & F1 $\times F 2$ & 0.48 & NS & & 0.05 & NS & \\
\hline
\end{tabular}


Table.4 Grain and straw yield $\left(\mathrm{kg} \mathrm{ha}^{-1}\right)$ of aerobic rice as influenced by weed management Practices under different seeding methods

\begin{tabular}{|c|c|c|c|c|c|c|c|}
\hline \multirow[t]{2}{*}{$\mathbf{T}$} & \multirow[t]{2}{*}{ Weed management practices } & \multicolumn{3}{|c|}{ Grain yield } & \multicolumn{3}{|c|}{ Straw yield } \\
\hline & & $\mathbf{S}_{1}$ & $\mathbf{S}_{\mathbf{2}}$ & Mean & $\mathbf{S}_{1}$ & $\mathbf{S}_{2}$ & Mean \\
\hline $\mathbf{T}_{1}$ & $\begin{array}{l}\text { Pretilachlor } 0.75 \mathrm{~kg} \text { ai } \mathrm{ha}^{-1} \text { as PE fb } \\
\text { Metsulfuron methyl }+ \text { Chlorimuron } \\
\text { ethyl } 4 \mathrm{~g} \text {. ai ha } \mathrm{ha}^{-1} \text { as PoE }+ \text { Cyhalofop } \\
\text { butyl } 75 \mathrm{~g} \text {. ai ha } \mathrm{ha}^{-1} \text { as PoE at } 15-20 \text { DAS. }\end{array}$ & 2357 & 2978 & 2668 & 2963 & 3648 & 3305.6 \\
\hline $\mathbf{T}_{2}$ & $\begin{array}{l}\text { Pretilachlor } 0.75 \mathrm{~kg} \text { ai } \mathrm{ha}^{-1} \text { as PE fb } \\
\text { Azimsulfuron } 35 \mathrm{~g} \text {.ai } \mathrm{ha}^{-1}+\text { Cyhalofop } \\
\text { butyl } 75 \mathrm{~g} \text {. ai ha }{ }^{-1} \text { as PoE } 15-20 \text { DAS. }\end{array}$ & 2630 & 3135 & 2883 & 3330 & 3860 & 3595.0 \\
\hline $\mathbf{T}_{3}$ & $\begin{array}{l}\text { Pretilachlor } 0.75 \mathrm{~kg} \text { ai } \mathrm{ha}^{-1} \text { as PE fb } \\
\text { Pyrazosulfuron ethyl } 20 \text { g.ai } \mathrm{ha}^{-1}+ \\
\text { Cyhalofop butyl } 75 \mathrm{~g} \text {. ai ha }{ }^{-1} \text { as } \mathrm{PoE} \text { at } \\
\text { 15-20 DAS. }\end{array}$ & 2330 & 3245 & 2787 & 2893 & 3814 & 3353.9 \\
\hline $\mathbf{T}_{4}$ & $\begin{array}{l}\text { Bispyribac sodium } 25 \mathrm{~g} \text { ai ha } \mathrm{h}^{-1} \text { as early } \\
\text { PoE fb 2-4-D } 0.5 \mathrm{~kg} \text {.ai ha }{ }^{-1} \text { at } 40 \text { DAS. }\end{array}$ & 2217 & 2978 & 2598 & 2868 & 3584 & 3226.2 \\
\hline $\mathbf{T}_{5}$ & $\mathrm{~T}_{1} \mathrm{fb}$ Hand weeding at $50 \mathrm{DAS}$ & 2658 & 3643 & 3150 & 3349 & 4433 & 3891.3 \\
\hline $\mathbf{T}_{6}$ & $\mathrm{~T}_{2} \mathrm{fb}$ Hand weeding at $50 \mathrm{DAS}$. & 2701 & 3735 & 3218 & 3486 & 4525 & 4005.6 \\
\hline $\mathbf{T}_{7}$ & $\mathrm{~T}_{3} \mathrm{fb}$ Hand weeding at $50 \mathrm{DAS}$. & 2546 & 3622 & 3084 & 3431 & 4411 & 3921.0 \\
\hline $\mathbf{T}_{8}$ & $\mathrm{~T}_{4} \mathrm{fb}$ Hand weeding at 50 DAS. & 2557 & 3449 & 3003 & 3338 & 4240 & 3789.5 \\
\hline $\mathbf{T}_{9}$ & Hand weeding at $20,40,60$ DAS & 2989 & 4064 & 3526 & 3755 & 4803 & 4279.3 \\
\hline $\mathbf{T}_{10}$ & Unweeded (control) & 958 & 1051 & 1005 & 1546 & 1637 & 1592.1 \\
\hline & Mean & 2366.1 & 3161.1 & & 3096.2 & 3895.7 & \\
\hline & & SEm \pm & CD & & SEm \pm & CD & \\
\hline & F1 & 44.73 & 128.09 & & 48.84 & 139.87 & \\
\hline & F2 & 100.0 & 286.4 & & 109.22 & 312.7 & \\
\hline & F1 $1 \times 2$ & 141.4 & 405.0 & & 154.46 & N.S. & \\
\hline
\end{tabular}

Interaction effect was significant with respect to number of panicles $\mathrm{m}^{-2}$ and found that more number of panicles were recorded with hand weeding thrice (at 20, 40 and $60 \mathrm{DAS})\left(\mathrm{T}_{9}\right)$ in line sowing method (288) than the broadcasting (251.3) which might be due to the effective control of weeds results in better performance of crop and more number of tillers per plant and produced more number of panicles.

Test weight (g), Panicle length (cm), Panicle weight $(g)$

Among seeding methods higher test weight, longer panicles, more panicle weight were observed with $\left(\mathrm{S}_{2}\right)$ line sowing (20.8), (17.4) and (1.28) over broadcasting $\left(\mathrm{S}_{1}\right)$ (18.9), (16.2) and (1.19) respectively, similar results were observed with Anwar et al., (2011). Among weed management practices, significantly higher test weight, longer panicles and more panicle weight were associated with hand weeding $\mathrm{T}_{9}$ (21.38), (20.1) and (1.43) which was followed by $\mathrm{T}_{6}$ (21.6), (17.3) and (1.34) respectively., and which in turn on par with $\mathrm{T}_{7}$, $\mathrm{T}_{5}, \mathrm{~T}_{8}$ which are significantly superior over other treatments, which might be resulted from weed free environment at the time of panicle production. Similar results were also reported by Thimme Gowda et al., (2009), Gopinath et al., (2012), Walia et al., (2009) (Table 3). $\mathrm{T}_{2}$ treatment found to be next best weed management practice in producing longer panicles followed by $T_{1}, T_{3}$, and $T_{4}$ which were at par themselves, recorded significantly longer panicles than un-weeded check $\left(\mathrm{W}_{10}\right)$. However, test weight, panicle length and panicle weight were not significantly influenced by the 
interaction effect of seeding methods and weed management practices.

\section{Yield}

Effect of seeding methods and weed management practices on grain yield of aerobic rice was analysed statistically and presented in this table 4. Among the seeding methods the more grain yield was recorded with line sowing $\left(\mathrm{S}_{2}\right)$ (3161) and was significantly superior over broadcasting $\left(\mathrm{S}_{1}\right)$ (2366) which might be due to less competition between crop and weeds and high yield attributes recorded with line sowing. Similar results were reported by Anwar et al., (2011), Moorthy and Rao (1991). The grain yield was significantly influenced by the interaction of seeding methods and weed management practices. The significantly highest grain yield (3526 kg ha-1) was obtained by hand weeding at 20, 40 and 60 DAS with the line sowing it might be due to the effective control of weeds under line sowing with hand weeding.

In conclusion, Sequential application of pre and post-emergence herbicides viz., azimsulfuron or pyrazosulfuron ethyl, chlorimuron ethyl+ metsulfuron methyl, bispyribac sodium or 2, 4$\mathrm{D}$ along with one hand weeding was found to be efficient weed control practice for getting more grain yield while it was with line sowing as suitable seeding method for getting higher grain yield in aerobic rice.

\section{References}

Anwar, P., Juraimi, A.S., Putech, A., Selamat, A., Man, A and Hakim, A. 2011. Seeding method and rate influence on weed suppression in aerobic rice. African Journal of Biotechnology.10 (68).

Carriger, F and Vallee, D. 2007. More crop per drop. Rice Today. 6 (2): 10-13.

Gopinath, K.A. Mina, B.L. Singh, K.P. and Nataraja, K.C. 2012. Integrated weed management in direct-seeded rainfed rice (Oryza sativa). Indian Journal of Agronomy. 57 (3): 245-249.

Moorthy, B.T.S and Rao, K.S. 1991. Influence of method of stand establishment and cultural and weed-control practices on performance of rainfed lowland rice (Oryza sativa) in coastal Orissa. Indian Journal of Agricultural Sciences. 61(8): 588-90.

Oerke, E.C and Dehne, H.W. 2004. Safeguarding production losses in major crops and the role of crop protection. Crop protection. 23: 275-285.

Saha, S. 2005. Evaluation of some new herbicide formulations alone or in combination with hand weeding in direct sown rainfed lowland rice. Indian Journal of Weed Science. 37 (1 and 2):103-104.

Thimmegowda, P., Shankaraiah, C., Jnanesh, A.C., Govindappa, M and Murthy, K.N. 2009. Studies on chemical weed control in aerobic rice (oryza sativa L.). Journal of Crop and Weed. 5(1): 320-323.

Walia, U.S., Bhullar, M.S., Nayyar, S and Walia, S.S. 2008b.Control of complex weed flora of dry seeded rice with preand post-emergence herbicides. Indian Journal of Weed Science. 40(3 and 4): 161-164.

\section{How to cite this article:}

Prashanthi, C.H., P. Laxminarayana, G.E.C.H. Vidyasagar and Harish Kumar Sharma, S. 2017. Yield Parameters and Yield of Aerobic Rice (Oryza sativa) as Influenced by Different Seeding Methods and Weed Control Measures. Int.J.Curr.Microbiol.App.Sci. 6(7): 2474-2480. doi: https://doi.org/10.20546/ijcmas.2017.607.351 